\title{
Efeito da Alteração Ambiental sobre Componentes Psicológicos e Parâmetros Fisiológicos durante Corrida em Atletas
}

\author{
Priscila Garcia Marques da Rocha ${ }^{1}$ \\ José Luiz Lopes \\ Solange Marta Franzói de Moraes \\ Universidade Estadual de Maringá
}

\begin{abstract}
RESUMO - Avaliou-se o efeito da alteração ambiental no afeto, percepção subjetiva do esforço e em parâmetros fisiológicos durante a corrida em atletas. Dezoito atletas de handebol atenderam a quatro sessões experimentais de corrida na esteira (linha de base, fragmentada, superestimada e indefinida) de 20 minutos. Foram avaliados: afeto, percepção subjetiva do esforço, frequência cardíaca e cortisol. Manipulou-se a informação sobre a duração da corrida. Verificou-se um aumento estatisticamente significativo para o afeto no decorrer da corrida das sessões "fragmentada" e "superestimada", assim como no nível de cortisol salivar aos 11 minutos da corrida. O controle do comportamento afetivo dos atletas parece amenizar desgastes fisiológicos frente a adversários desconhecidos e condições indefinidas de jogo.
\end{abstract}

Palavras-chave: atletas; afeto; percepção subjetiva do esforço.

\section{Effect of Environment Alteration in Psychological Components and Physiological Parameters during the Treadmill Running in Athletes}

\begin{abstract}
It was evaluated the effect of environmental alteration in affect, perceived exertion and in physiological parameters during treadmill running in athletes. Eighteen handball players participated in four running experimental sessions (baseline, fragmented overrated and unknown) for 20 minutes. It was analyzed: affect, rating of perceived exertion, heart rate and cortisol. Information about the running duration was manipulated. It was observed a statistically significant increase in affect during the treadmill running for the "fragmented" and "overrated " sessions, as well as in salivary cortisol level at the 11 th minute of running. The control of athletes' affective behavior seems to relieve physiological damage when facing unknown opponents and uncertain game conditions.
\end{abstract}

Keywords: athletes; affect; rating of perceived exertion.

A psicologia do esporte e do exercício é uma área de investigação em expansão. Sua relevância se baseia, dentre outras aspectos, nos achados sobre os efeitos psicológicos e psicossomáticos decorrentes da prática regular de exercícios, seja com objetivos de lazer e saúde, seja no contexto de esportes com alto rendimento (Weinberg \& Gould, 2008). Evidências importantes na conduta de atletas pré, durante e pós-jogo, por exemplo, demonstram que quando um patamar físico ótimo é alcançado, em situações em que a tática de jogo é equivalente entre adversários, o fator decisivo para a vitória consiste em como o atleta percebe e se comporta frente ao ambiente competitivo.

A psicologia do esporte e do exercício, em conjunto com a fisiologia do esforço, dispõe de meios de mensuração adaptada à prática esportiva que visam aferir como o atleta se percebe fisiologicamente e afetivamente na conjuntura de sua rotina de treinamento/competição. Sendo assim, a percepção subjetiva do esforço (PSE) tem sido considerada na mensuração da intensidade do exercício físico como um componente especificamente fisiológico,

1 Endereço para correspondência: Rua Luiz Fabeni, 35, Jardim Custódio. Marialva, PR. CEP 86 990-000. Telefone: (44) 3232 5792. E-mail: pgmrocha@yahoo.com.br. sendo avaliadas variáveis importantes para o esforço como a força muscular, capacidade cardiovascular, concentração de metabólitos, entre outras (Baden, McLean, Tucker, Noakes \& St Clair Gibson, 2005). No entanto, alguns estudos demonstraram que determinados parâmetros psicológicos, como o estado afetivo, podem também influenciar a percepção de esforço (Baden \& cols., 2005; Hardy \& Rejeski, 1989; Parfitt, Markland \& Holmes, 1994; Parfitt, Rose \& Markland, 2000).

Como sugerido por Damásio (1996), o afeto é capaz de provocar um mapeamento cerebral inconsciente do estado fisiológico do corpo (Hipótese do Marcador Somático). Isso pode ocorrer quando o afeto se manifesta na sua dimensão de curta duração (estado de humor), quando comportamentos psicológicos não demonstrados publicamente (sentimentos) ou quando o sujeito desconhece ou não pode prever como um fator ambiental externo se desencadeará. Modificações nesse mapeamento fisiológico decorrente das consequências psicológicas podem ocorrer, gerando alterações afetivas. A hipótese do marcador somático, de acordo com Lambert, St Clair Gibson e Noakes (2004), pode interferir na resposta ao esforço físico, assim como na percepção subjetiva do esforço (Baden \& cols., 2005). $\mathrm{O}$ conhecimento das plenas condições de exercício físico, 
como a duração do esforço, também tem sido apontado como um fator de controle importante dos aspectos psicológicos e fisiológicos (Hardy \& Rejeski, 1989; Kang \& cols., 2003).

Os estudos citados sugerem que tanto as dimensões afetiva e fisiológica quanto a ecológica interferem na percepção subjetiva do esforço. Por essa razão, este estudo se propôs a avaliar o efeito da alteração da duração da corrida sobre o afeto, a percepção subjetiva do esforço e alguns parâmetros fisiológicos (frequência cardíaca e cortisol) em atletas.

\section{Método}

\section{Participantes}

A amostra selecionada para este estudo foi composta por atletas de handebol, da categoria adulta. Participaram do estudo 18 atletas ( 9 homens e 9 mulheres) que concordaram com as exigências do estudo. Os atletas foram esclarecidos sobre o objetivo do estudo, os procedimentos, os testes e os protocolos que seriam utilizados. Após o fornecimento dessas informações, os participantes assinaram voluntariamente o termo de consentimento livre e esclarecido. O presente projeto foi aprovado pelo Comitê de Ética em Pesquisa com Seres Humanos da Universidade Estadual de Maringá.

\section{Procedimentos}

O procedimento experimental compreendeu uma sessão de teste funcional, uma sessão de familiarização e quatro sessões de corrida na esteira (linha de base, fragmentada, superestimada e indefinida), em que a informação sobre o tempo de corrida foi a variável manipulada.

A sessão de teste funcional consistiu em submeter os atletas ao teste ergométrico na esteira, em que a velocidade e a inclinação da esteira aumentaram de forma intervalar, com monitoração da frequência cardíaca e da pressão arterial. O objetivo era avaliar se as condições da saúde cardiovascular permitiriam a execução das sessões experimentais com total segurança pelos atletas. O teste funcional é um procedimento padrão em fisiologia do exercício (ACSM, 2007). Nessa sessão, os atletas alcançaram individualmente a máxima velocidade de corrida na esteira para estabelecer $70 \%$ da velocidade de pico encontrada para realização das quatro sessões experimentais de corrida.

Após a sessão de teste funcional, oportunizou-se aos participantes uma sessão de familiarização, a qual tinha os seguintes objetivos: proporcionar aos atletas o conhecimento dos protocolos utilizados nas triagens experimentais; habituar o atleta com a esteira de corrida; e experimentar a velocidade de corrida estabelecida para as triagens experimentais.

Posteriormente, foram aplicadas quatro sessões experimentais: na primeira sessão foi aplicado o Tratamento A (linha de base) e nas três posteriores foi realizada a aplicação cega e aleatória dos tratamentos B, C e D, conforme descrito a seguir:

Tratamento A (Linha de Base): Corrida de 20 minutos de duração, com conhecimento completo da duração da corrida. Ou seja, antes do início do teste o atleta era informado que a duração da corrida seria de 20 minutos;

Tratamento $B$ (Fragmentada): Corrida de 20 minutos, com conhecimento fragmentado da duração da corrida. Isto é, o atleta era informado inicialmente que a prova teria a duração de 10 minutos e, ao $10^{\circ}$ minuto, recebia a informação de que haveria 10 minutos adicionais de corrida;

Tratamento $C$ (Superestimada): Corrida de 20 minutos, com informação prévia de 10 minutos de corrida, e ao $10^{\circ}$ minuto, nova informação de 20 minutos adicionais de corrida, mas com acréscimo real de apenas 10 minutos;

Tratamento D (Indefinida): Corrida de 20 minutos, mas o atleta não era informado sobre a duração da corrida.

A coleta aconteceu no final do primeiro e no começo do segundo semestre do ano de 2007, no Laboratório de Fisiologia do Esforço do Departamento de Ciências Morfofisiológicas da Universidade Estadual de Maringá, por dois pesquisadores (um fisiologista e um profissional em educação física), com o acompanhamento de um psicólogo. A coleta durou cerca de 12 semanas.

\section{Instrumentos}

A percepção subjetiva do esforço foi mensurada por meio da Escala de Percepção Subjetiva do Esforço, proposta por Borg (2000). Essa escala é composta por valores de 0 a 10 , e para cada valor há seu correspondente em sensação física, compreendida entre muito fácil (0) a exaustivo (10). Os números de 0-10 são baseados na frequência cardíaca de 60-200 batimentos por minuto (bpm), sendo que o número 0 , que indica repouso na escala de Borg, corresponde à frequência cardíaca entre 60 e 80 bpm; o número 1 que indica exercício "muito leve", corresponde à frequência cardíaca entre 90 e 110 bpm, e assim sucessivamente até o número 10 , que indica exercício "exaustivo", com frequência cardíaca de 200 bpm. É considerada uma escala de percepção subjetiva porque o próprio indivíduo aponta a sensação física percebida. A forma de aplicação dessa escala foi explicada cuidadosamente durante a sessão de familiarização e antes de cada sessão de corrida.

Durante a corrida, o estado afetivo também foi avaliado. Para isso, utilizou-se a Escala de Afeto, de Hardy e Rejeski (1989), que avalia a valência afetiva em um momento específico. Essa é uma escala bipolar que contém um único item de componente afetivo, o qual indica como é percebido o afeto no exato momento em que o sujeito é questionado. $\mathrm{O}$ indivíduo deve descrever a classificação: + 5 (muito bem); + 3 (bem); + 1 (levemente bem); 0 (neutro); - 1 (levemente mal); - 3 (mal); + 5 (muito mal). Para essa avaliação, os atletas foram relembrados a cada sessão que a Escala de Afeto descreve se o exercício está 
prazeroso ou desagradável, e não a descrição da intensidade do esforço.

As duas escalas descritas acima foram aplicadas em todas as sessões experimentais, com frequência de 11 vezes em cada sessão, mais precisamente, no $3^{\circ}, 5^{\circ}, 8^{\circ}, 9^{\circ}, 10^{\circ}, 11^{\circ}$, $14^{\circ}, 17^{\circ}, 19^{\circ}$ e $20^{\circ}$ minuto. Utilizou-se uma cópia da escala ampliada, para que os sujeitos pudessem visualizá-la com facilidade a $1 \mathrm{~m}$ de distância.

A frequência cardíaca foi mensurada durante todas as sessões experimentais, por meio de eletrocardiógrafo digital. A ergometria foi realizada pelo $\operatorname{ErgoPC} 13 \AA$ para Windows ${ }^{\circledR}$, da Micromed $\AA$. Os parâmetros hemodinâmicos (pressão arterial sistólica e diastólica e frequência cardíaca máxima) foram controlados em todas as triagens experimentais.

Para as medidas do nível de cortisol, a primeira amostra de saliva foi coletada em condições basais, antes da primeira sessão experimental, seguindo os seguintes critérios: coletar até 2 horas depois de acordar; estar em jejum; não ingerir nenhuma bebida (com exceção de água) por 30 minutos antes da coleta; permanecer em repouso 1 hora antes da coleta; lavar a boca com água imediatamente antes da coleta, com bochechos leves; não coletar a saliva em caso de lesões orais com sangramento; não ter feito tratamento dental 24 horas antes da coleta; não escovar os dentes nas últimas 3 horas, para evitar sangramento gengival. A coleta foi realizada com um algodão colocado sob a língua durante 2 a 3 minutos, retornando o algodão em seguida ao túbulo plástico de $20 \mathrm{ml}$.

Nas sessões experimentais, o nível de cortisol foi medido a partir de amostras de saliva no $4^{\circ}, 11^{\circ}$ e no $20^{\circ}$ minuto de esforço. Os participantes foram instruídos a evitar o consumo de cafeína, álcool e similares, e a prática de atividades físicas intensas, nas 12 horas antecedentes à coleta de dados. Para a preparação da coleta da amostra de saliva, os sujeitos foram instruídos a fazer lavagem bucal com água por 1 minuto, com o objetivo de remover substâncias como a clorina, a qual mascara os resultados dos níveis de cortisol na saliva.

Logo após a coleta, as amostras foram congeladas a $-20^{\circ} \mathrm{C}$. Para análise do cortisol, utilizou-se o kit Active Cortisol EIA $\mathbb{}$ (for saliva) (Diagnostic System Laboratories Inc, Beckman Coulter Company, Texas, USA).

\section{Análise de dados}

A análise de variância two-way foi utilizada para comparar as triagens experimentais subsequentes (linha de base, fragmentada, superestimada e indefinida). O nível de $\alpha$ estabelecido correspondeu a $\mathrm{p} \leq 0,05$ para valores estatisticamente significativos.

\section{Resultados}

Esta seção adota a seguinte estrutura organizacional: a descrição das modificações nas respostas fisiológicas relacionadas à frequência cardíaca, ao cortisol e à percepção subjetiva do esforço e, em seguida, a apresentação dos escores de afeto em relação à manipulação da informação verbal da duração da corrida.

A Figura 1 demonstra a frequência cardíaca dos atletas durante as sessões experimentais de corrida. A frequência cardíaca variou de forma homogênea entre as quatro sessões, não sendo encontradas diferenças estatisticamente significativas entre elas.

Os níveis de cortisol salivar dos atletas estão demonstrados na Tabela 1. Nota-se aumento estatisticamente significativo do nível de cortisol no $11^{\circ}$ minuto de corrida para as sessões experimentais "fragmentada" e "superestimada" quando comparadas à linha de base. $\mathrm{O} 11^{\circ}$ minuto de corrida correspondeu ao $1^{\circ}$ minuto posterior à retificação da informação sobre a duração do esforço.

A Figura 2 apresenta a percepção subjetiva do esforço dos atletas durante as sessões experimentais. A sessão experimental "indefinida" apresentou os menores níveis na percepção subjetiva do esforço, sendo os níveis obtidos entre o $8^{\circ}$ e o $12^{\circ}$ minutos de corrida estatisticamente diferentes daqueles observados na "linha de base". No entanto, a partir do $14^{\circ}$, a percepção subjetiva do esforço nessa sessão elevou-se, equiparando-se às das demais sessões até o final da corrida.

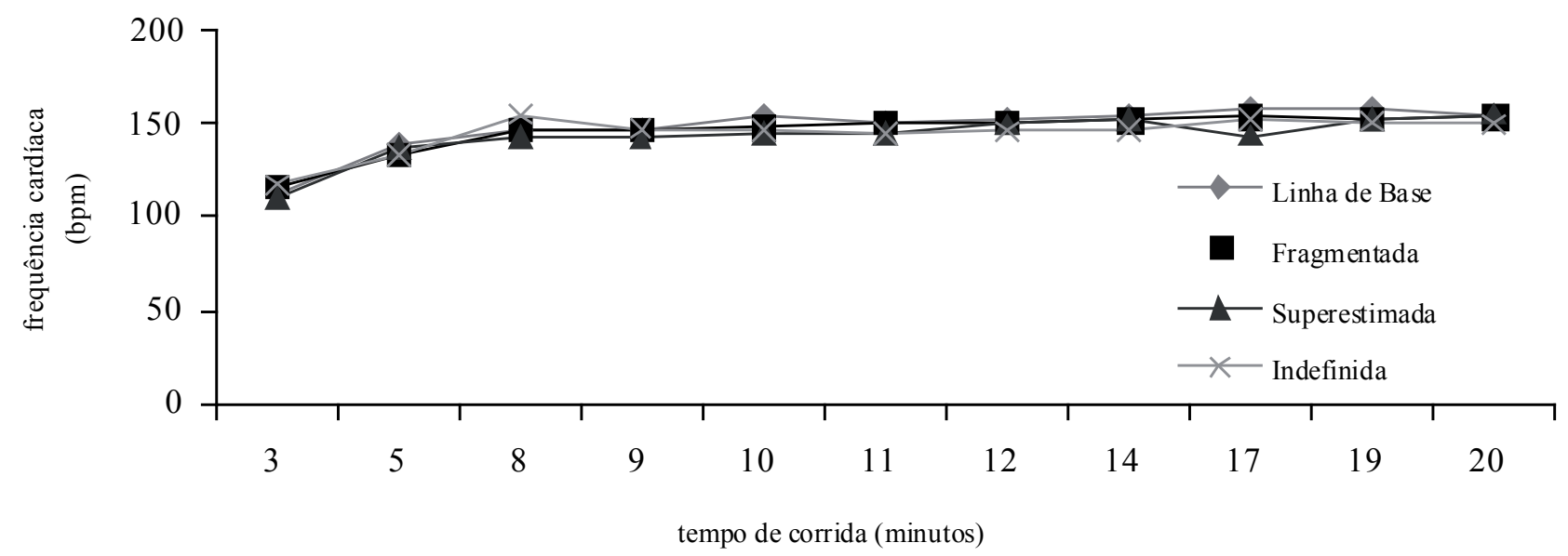

Figura 1. Frequência cardíaca dos atletas durante a corrida nas sessões experimentais ("linha de base", "fragmentada", "superestimada" e "indefinida"). 
Tabela 1. Nível de cortisol da saliva (nmol/l) dos atletas nas quatro triagens experimentais.

\begin{tabular}{|c|c|c|c|c|c|c|c|c|c|c|c|c|}
\hline & \multicolumn{3}{|c|}{ Linha de Base } & \multicolumn{3}{|c|}{ Fragmentada } & \multicolumn{3}{|c|}{ Superestimada } & \multicolumn{3}{|c|}{ Indefinida } \\
\hline & M & & $\mathrm{DP}$ & M & & $\mathrm{DP}$ & M & & DP & M & & DP \\
\hline $3 \mathrm{~min}$ & 10,50 & \pm & 2,76 & 10,19 & \pm & 3,42 & 9,53 & \pm & 1,41 & 9,19 & \pm & 1,81 \\
\hline $11 \mathrm{~min}$ & 9,26 & \pm & 1,54 & $* 11,59$ & \pm & 1,92 & $* 10,69$ & \pm & 1,48 & 9,96 & \pm & 2,58 \\
\hline $18 \mathrm{~min}$ & 9,56 & \pm & 1,56 & 9,89 & \pm & 3,04 & 6,96 & \pm & 2,23 & 9,43 & \pm & 2,55 \\
\hline
\end{tabular}

Nota: $\mathrm{M}=$ média; $\mathrm{DP}=$ desvio padrão; $* \mathrm{p}<0,05$.

Não foram encontradas diferenças estatisticamente significativas entre as demais triagens.

A Figura 3 apresenta os escores de afeto dos atletas de handebol durante as sessões experimentais. Foram observadas diferenças estatisticamente significativa apenas nas sessões experimentais "fragmentada" e "superestimada", nos minutos subsequentes ao $10^{\circ}$ minuto, quando a informação sobre a duração da corrida foi verbalmente manipulada.

\section{Discussão}

Destaca-se, neste estudo, o importante papel da percepção que o atleta tem do seu ambiente, representado aqui pela informação sobre o tempo de corrida, e a influência de aspectos psicológicos sobre as respostas fisiológicas durante o exercício físico. A percepção subjetiva do esforço, comumente entendida como componente do campo fisiológico (Cafarelli, 1982; Kang \& cols, 2003; Robertson, 1982) e características endócrinas, representadas neste estudo pelo cortisol, parecem não ser características fisiológicas isoladas ou independentes do contexto psicológico.

Em virtude de ter-se obtido frequência cardíaca similar e homogênia entre as sessões experimentais, comprova-se que a intensidade do esforço foi a mesma para todas as triagens; e, subjacente a isto, variáveis hemodinâmicas dos atletas (pressão arterial), climatização e movimenta- ção no laboratório foram rigorosamente controladas. Por essa razão, de acordo com Baden e cols (2005) e St Clair Gibson e cols. (2003), a fisiologia do esforço isoladamente não encerra explicações justificáveis para muitos comportamentos fisiológicos durante o exercício, como a alteração do cortisol logo após a alteração da informação sobre o tempo de duração de corrida (triagens "fragmentada" e "superestimada").

Adjacente a isto, logo após a informação da duração da corrida ser alterada ao $10^{\circ}$ minuto para as sessões "fragmentada" e "superestimada", foram encontradas coincidências interessantes nos escores de afeto e o nível de cortisol. Os atletas receberam a informação sobre a duração da corrida antes de começar a correr nas sessões. Em especial, as triagens "fragmentada" e a "superestimada" agregaram semelhanças na informação inicial sobre a duração da corrida para o atleta, que foi de 10 minutos. Os argumentos de Hardy e Rejeski (1989) e Baden, Warwick-Evans e Lakomy (2004) sobre a capacidade que uma pessoa tem de antecipar estratégias de corrida diante de uma informação pré-existente sugerem que os atletas se prepararam para correr por 10 minutos, sendo que paralelo a isto, o nível de cortisol estava baixo e os atletas relatavam sentir-se "bem" e "muito bem" durante este tempo inicial de esforço. No entanto, no decorrer da corrida, a informação foi retificada, aumentando o tempo de esforço, o que, por sua vez, pareceu causar impacto no comportamento afetivo do atleta, como é possível notar pelo súbito aumento nos níveis de cortisol neste ponto

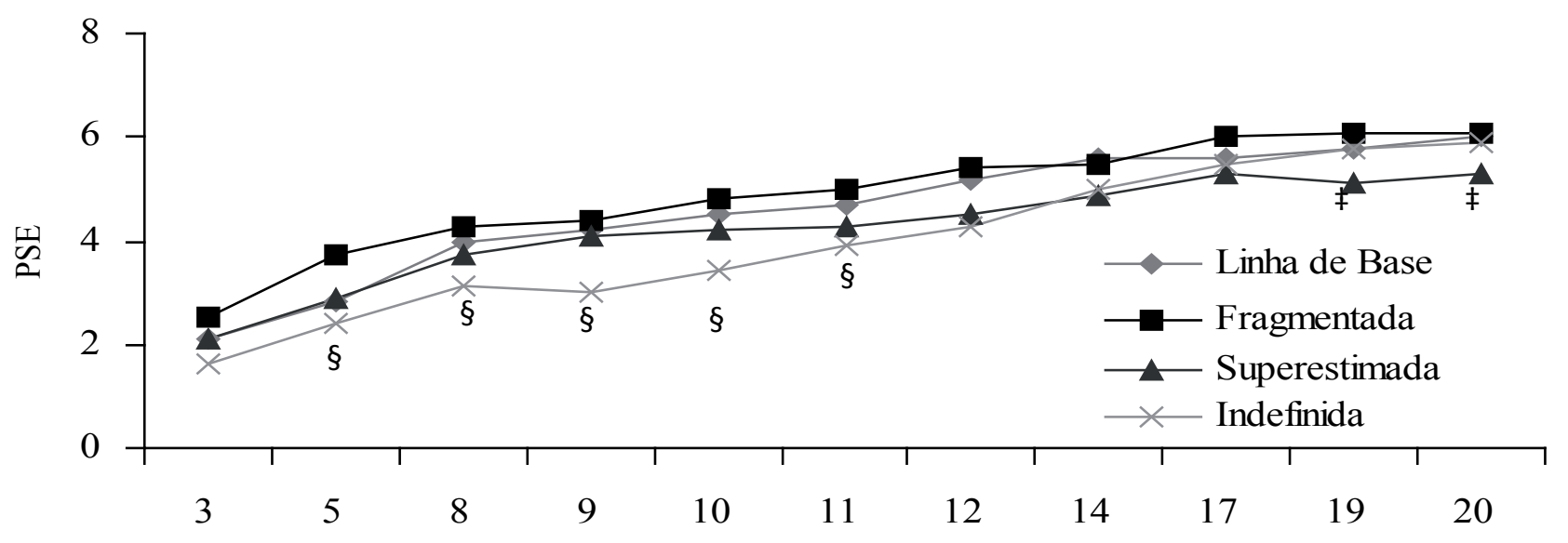

tempo de corrida (minutos)

Figura 2. Percepção subjetiva do esforço dos atletas durante as sessões experimentais, sendo †๋ para diferenças estatisticamente significativas entre a "linha de base" e a sessão "fragmentada" e § para diferenças estatisticamente significativas entre a "linha de base" e a sessão "indefinida". 


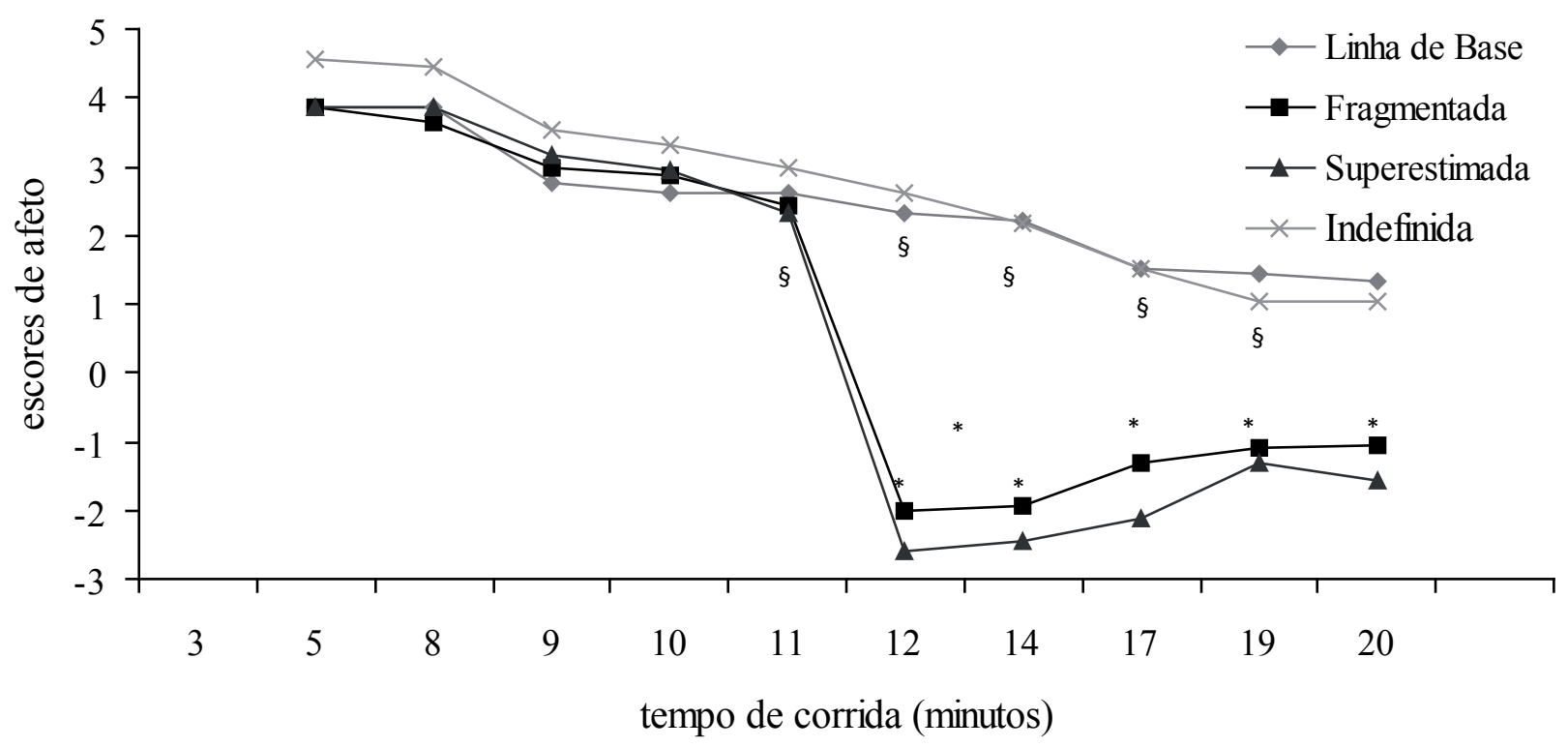

Figura 3. Sentimentos dos atletas durante as sessões experimentais, sendo § para diferenças estatisticamente significativas entre a "linha de base" e a sessão "fragmentada", e * para diferenças estatisticamente significativas entre a "linha de base" e a sessão "superestimada".

temporal da corrida e nos escores de afeto, tendo os atletas relatado sentir-se "mal" de repente.

O cortisol pode causar mudanças no estado afetivo, inclusive durante o exercício físico. É um hormônio catabólico, que aumenta a disponibilidade energética no sangue, assim como as funções simpáticas (Urhousen, Gabriel \& Kindermann, 1998). No entanto, os mecanismos do cortisol durante o exercício físico ainda não são conclusivos. Estados afetivos negativos envolvem mudanças fisiológicas, com respostas hormonais, aumento da temperatura muscular e ventilação respiratória, que podem ser mensuráveis. Todavia, a possibilidade de medir a resposta fisiológica pode mascarar o papel do afeto e da real influência psicológica em resposta ao esforço físico (St Clair Gibson \& cols., 2003).

Quando os atletas não receberam a informação da duração da corrida na sessão experimental "indefinida", a percepção subjetiva do esforço foi mais baixa (os atletas se sentiram menos cansados) do que nas demais triagens, pressupondo que o atleta pode antecipar o comportamento durante a corrida, diante de situações diferenciadas de informação sobre o esforço físico. Esse fato corrobora os achados de Baden e cols. (2005), que concluiram que quando a informação da corrida é alterada ou desconhecida e a antecipação que o atleta faz antes de começar a correr se diferencia do esforço "real" que teria que realizar, a percepção subjetiva do esforço se modifica de acordo com a situação ambiental imposta ao atleta.

De acordo com Parfitt, Markland e Holmes (1994), a percepção subjetiva do esforço não é adequada para experiência subjetiva com o exercício físico, no entanto, os escores de afeto sim. Foi possível notar, no presente estudo, que quanto maior a percepção subjetiva do esforço, menor foi o escore afetivo. Esses resultados corroboram aqueles de Hardy e Rejeski (1989), Parfitt, Markland e Holmes (1994), Parfitt, Rose e Markland (2000), cujas conclusões afirmam que a percepção subjetiva do esforço e a escala de afeto não possuem sensibilidade na mesma proporção para avaliar a influência psicológica sobre o esforço físico; no entanto, o afeto (sentimentos) do atleta pode alterar a percepção subjetiva do esforço.

\section{Considerações Finais}

A partir dos resultados encontrados, e tendo como objetivo a aplicação prática das conclusões desta pesquisa em um contexto real da relação técnico-atleta em uma situação de competição, elaboramos as seguintes suposições: (1) O técnico, visando minimizar o desgaste psicológico dos atletas frente a um adversário que pode surpreender durante o jogo (sessão "fragmentada") ou frente a um adversário superior (sessão "superestimada"), poderia esperar de seus atletas os seguintes parâmetros fisiológicos: frequência cardíaca inalterada, aumento no nível de cortisol conforme ocorre o embate; assim como redução do bem-estar do atleta durante o jogo e consequente aumento na percepção subjetiva do esforço; (2) O técnico, visando minimizar o desgaste psicológico dos atletas frente a um adversário indefinido ou desconhecido, poderia esperar nenhuma alteração nos parâmetros fisiológicos; e, quanto aos componentes psicológicos, poderia esperar melhor controle emocional relacionado ao afeto e, assim, percepção subjetiva do esforço mais baixa.

Devido ao fato dos ambientes laboratoriais dificultarem a simulação de situações psicológicas, sugere-se a realização de um estudo similar a este no ambiente ecológico do atleta. Por mais próximo que seja o estímulo laboratorial à situação real, o ambiente ecológico do atleta ou do indivíduo que pratica exercícios físicos pode, de forma mais complexa, apresentar a interpolação entre respostas fisiológicas e psicológicas. Isso porque a interação que o atleta, como sujeito de características próprias, faz com o ambiente esportivo pode influenciar de forma ainda mais profunda esta relação contínua entre os parâmetros fisiológicos e os componentes psicológicos. 


\section{Referências}

American College of Sports Medicine - ACSM (2007). Diretrizes do ACSM para os testes de esforços sua prescrição $\left(7^{\mathrm{a}}\right.$ ed.) (G. Taranto, Trad.). Niterói: Guanabara Koogan. (Trabalho original publicado em 1975)

Baden, D. A., McLean, T. L., Tucker, R., Noakes, T. D., \& St Clair Gibson, A. (2005). Effect of anticipation during unknown or unexpected exercise duration on rating of perceived exertion, affect, and physiological function. British Journal of Sports Medicine, 39, 742-746.

Baden, D. A., Warwick-Evans, L., \& Lakomy, J. (2004). Am I nearly there? The effect of anticipated running distance on perceived exertion and attentional focus. Journal of Sport \& Exercise Psychology, 26, 215-231.

Cafarelli, E. (1982). Peripheral contributions to the perceived effort. Medicine Science in Sports and Exercise, 14, 382-389.

Damasio, A. R. (1996). O erro de Descartes: emoção, razão e cérebro humano. São Paulo: Companhia das Letras.

Hardy C. J., \& Rejeski, W. J. (1989). Not what, but how one feels: The measurement of affect during exercise. Journal of Sports and Exercise Psychology, 11, 304-317.

Kang, J, Hoffman, J. R, Walker, H., Chaloupka, E. C, \& Utter, A. C. (2003). Regulating intensity using perceived exertion during extended exercise periods. European Journal of Applied Physiology, 89, 475-482.

Lambert, E. V., St Clair Gibson, A., \& Noakes, T. D. (2004). Complex system model of fatigue: Integrative homoeostatic control of peripheral physiological systems during exercise in humans. British Journal of Sports Medicine, 39, 52-64.
Parfitt, G., Markland, D., \& Holmes, C. (1994). Responses to physical exertion in active and inctive males and females. Journal of Sport \& Exercise Psychology, 16, 178-186.

Parfitt, G., Rose, E. A., \& Markland, D. (2000). The effect of prescribed and preferred intensity exercise on psychological affect and the influence of baseline measures of affect. Journal of Health Psychology, 5, 231-240.

Robertson, R. J. (1982). Central signals of perceived exertion during dynamic exercise. Medicine Science in Sports and Exercise, 14, 390-396.

St Clair Gibson, A., Baden, D. A., Lambert, M. I., Lambert, E. V., Harley, Y. X. R., Hampson, D., Russel, V. A., \& Noakes, T. D. (2003). The conscious perception of the sensation of fatigue. Sports Medicine, 33, 116-176.

Urhausen, A., Gabriel, H., \& Kindermann, W. (1998). Impaired pituitary hormonal response to exhaustive exercise in overtrained endurance athletes. Medicine in Science \& Sports \& Exercise, 30, 407-414.

Wienberg, R. S., \& Gould, D. (2008). Fundamentos da Psicologia do Esporte e do Exercício (4 ed.) (C. Monteiro, Trad.). Porto Alegre: Artmed. (Trabalho original publicado em 2001)

Recebido em 06.03.2009

Primeira decisão editorial em 24.09.2009

Versão final em 13.10.2009

Aceito em 22.10.2009 